\title{
Aspectos socioeconômicos e ambientais da produção de farinha de mandioca na comunidade quilombola Amazônica do Cuxiú, Bonito/PA
}

Este trabalho teve como principal motivação, conhecer os aspectos sociais, ambientais e econômicos, inerentes à produção da farinha de mandioca na Comunidade Quilombola do Cuxiú, Bonito-PA, Amazônia. Na tentativa de alcançar esse objetivo geral sob a visão holística da sustentabilidade, definiram-se cinco objetivos específicos: a) Verificar entraves e potencialidades na produção de farinha na comunidade quilombola; b) Acompanhar a dinâmica social do beneficiamento da mandioca, dentro do cotidiano quilombola; $c$ ) Identificar a forma de descarte de resíduos provenientes do beneficiamento da mandioca; e) Identificar aspectos econômicos da produção de farinha de mandioca. As ferramentas utilizadas para a coleta de dados fora

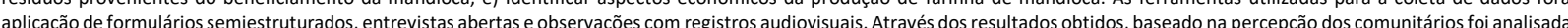
a importância da produção farinha como fator de reprodução social dos comunitários, bem como uma avaliação parcial dos aspectos ambientais referentes a atividade, pontuando possíveis a importância da produção farinha como fator de reprodução social dos comunitários, bem como uma avaliação parcial dos aspectos ambientais referentes a atividade, pontuando possíveis impactos ambientais e ainda conhecer a forma de comercialização da farinha de mandioca pelos comunitários. A atividade de farinha historicamente acompanhou o processo de implantação e desenvolvimento da comunidade, garantindo a subsistência no campo e ressaltando a solidariedade e as relações de mutualidade entre o povo quilombola. Fatores como a disponibilidade de emprego no campo para os comunitários e o baixo nível de evasão, caracterizam a comunidade como socialmente sustentável. Enquanto isso, a simbiose entre a comunidade e os recursos naturais demonstra o interesse das famílias em manter o ecossistema local preservado e a necessidade de aprimorar as formas de produção na lavoura, sobretudo para melhorar beneficiamento da farinha de mandioca. Portanto, a sustentabilda de farinha para aumentar a obtenção de renda e a qualidade da farinha, produto tão importante para o desenvolvimento sustentável regional das comunidades tradicionais

Palavras-chave: Farinha de Mandioca; Comunidades Tradicionais; Quilombos; Desenvolvimento Sustentável.

\section{Socioeconomic and environmental aspects of cassava flour production in the Amazon quilombola community of Cuxiú, Bonito/PA}

\begin{abstract}
This work had as main motivation, to know the social, environmental and economic aspects, inherent to the production of manioc flour in the Quilombola do Cuxiú Community, Bonito-PA Amazonia. In an attempt to a hieve this gen of flour in the quilombola community; b) Monitor the social dynamics of the processing of cassava, within the quilombola daily life; c) Identify how to dispose of residues from the processing of cassava; e) Identify economic aspects of cassava flour production. The tools used for data collection for the application of semi-structured forms, disseminated and disseminated with of cassava; e) Identify economic aspects of cassava flour production. The tools used for data collection for the application of semi-structured forms, disseminated and disseminated with
audiovisual records. Through the results obtained, based on the perception of those responsible for the analysis of the importance of flour production as a factor of social reproduction of audiovisual records. Through the results obtained, based on the perception of those responsible for the analysis of the importance of flour production as a factor of social reproduction of of cassava flour by the competent ones. The flour activity has historically accompanied the process of implantation and development of the community, guaranteeing subsistence in the countryside and emphasizing solidarity and mutual relations among the quilombola people. Factors such as the availability of employment in the field for the related and low level of evasion characterize the community as socially sustainable. Meanwhile, a symbiosis between the community and natural resources of interest to families in maintaining the preserved local ecosystem and the need to improve production methods in the field, especially to improve the processing of cassava flour. Therefore, economic sustainability needs to be improved through new market niches to sell the production of cassava flour and improvements in the infrastructure of flour houses to increase income and the quality of flour, a product so important for sustainable regional development traditional communities.
\end{abstract}

Keywords: Cassava flour; Traditional Communities; Quilombos; Sustainable development.

Topic: Uso Sustentável da Biodiversidade

Reviewed anonymously in the process of blind peer.
Received: 05/12/2020

Approved: $20 / 02 / 2021$
Fernanda Gisele Santos de Quadros

Universidade Federal Rural da Amazônia, Brasil

http://lattes.cnpq.br/7782947341657509

http://orcid.org/0000-0001-8062-4670

fgsquadros@gmail.com

Igor de Souza Gomide (D)

Universidade Federal Rural da Amazônia, Brasil

http://lattes.cnpq.br/2069490721355492

http://orcid.org/0000-0002-2703-2737

igor.gomide@ufra.edu.br
Referencing this:

QUADROS, F. G. S.; GOMIDE, I. S.. Aspectos socioeconômicos e ambientais da produção de farinha de mandioca na comunidade quilombola Amazônica do Cuxiú, Bonito/PA. Nature and Conservation, v.14, n.1, p.55-61, 2021. DOI: http://doi.org/10.6008/CBPC2318-2881.2021.001.0006 


\section{INTRODUÇÃO}

Povos e Comunidades tradicionais são grupos culturalmente diferenciados e que se reconhecem como tais, que possuem formas próprias de organização social, que ocupam e usam territórios e recursos naturais como condição para sua reprodução cultural, social, religiosa, ancestral e econômica, utilizando conhecimentos, inovações e práticas gerados e transmitidos pela tradição (BRASIL, 2007).

Brandão et al. (2012) comenta que a principal característica das populações e comunidades tradicionais, é a articulação de um modo de vida ancorado na dependência da natureza e mesmo na simbiose com os ciclos naturais que marcam a disponibilização ou escassez sazonal dos recursos naturais renováveis.

As comunidades remanescentes de quilombos são destacadas como uma forma bastante particular de organização produtiva no espaço rural, principalmente pela característica de apresentar uma área de produção de uso comum (SILVA et al., 2018). Fator que os caracterizam em comunidades tradicionais.

A constituição federal de 1988 foi um marco para a valorização dos direitos e valores quilombolas, por reconhece-los como parte do patrimônio cultural Brasileiro, que retratam e preservam a cultura afrobrasileira, remanescente do povo africano que colonizou este País, e, portanto, devem ser protegidas pelo Estado. Além disso, foi lhes assegurado o direito à propriedade definitiva das terras em que estejam ocupando, bem como a emissão dos títulos (BRASIL, 2016).

Uma característica marcante dos povos indígenas, caboclos, ribeirinhos e quilombolas, é o cultivo da mandioca, um dos principais componentes do cardápio dessas famílias de baixa renda (BARLOW et al., 2017).

No Brasil, são produzidas 20. 606.037 toneladas de mandioca em uma área de 1.407. 345 hectares. Sendo, 4. 234.597 toneladas produzidas em solo paraense, equivalente a $20,55 \%$ em relação à produção nacional, abrangendo uma área de 295, 137 hectares (IBGE, 2017).

De acordo com Souza et al. (2017) o estado do Pará destaca-se como o principal estado produtor de mandioca do Brasil. Ressalta-se que o cultivo da mandioca na região Norte, é conduzido basicamente por pequenos agricultores familiares em assentamentos agrícolas e comunidades tradicionais, tendo papel crucial na sua subsistência.

De modo geral, as etapas de produção da farinha podem ser definidas basicamente em: Plantio, colheita, transporte, descascamento, lavagem, trituração, prensagem, esfarelamento, torração, empacotamento e armazenagem (figura 1).

Ainda que seja uma atividade tradicional na região, a produção da farinha envolve alguns problemas a serem solucionados. Inserção de máquinas no plantio e colheita da mandioca, melhoramento das condições sanitárias das fábricas e adição de tecnologias em todas as fases do processamento: raspagem, ralação e secagem (FREITAS et al., 2011).

Outro fator relevante, diz respeito à tomada de preço da farinha de mandioca, pois muitos agricultores não têm noção de custos e muitas vezes não arrecadam nem o suficiente para custear os materiais de produção. Por esse motivo, produtos com pouco valor agregado resultam na baixa remuneração dos produtores (ARAÚJO, 2013). 


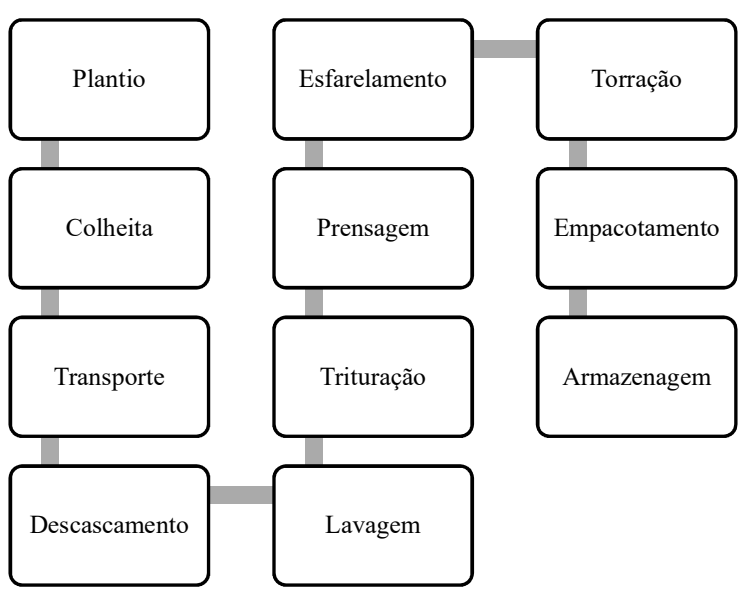

Figura 1: Etapas da produção de farinha. Fonte: Adaptado de Chiste (2011); SEBRAE (2006).

O processo de beneficiamento da farinha de mandioca gera o efluente conhecido como manipueira que significa dizer "o que brota da mandioca" no tupi-guarani. O líquido contém elevada concentração de matéria orgânica, carboidratos e cianeto, representando impacto negativo ao meio ambiente, principalmente quando despejado diretamente em mananciais e no solo (LIMA, 2010).

Assim, o objetivo deste estudo foi conhecer a dinâmica de uma comunidade tradicional quilombola e através de aspectos socioeconômicos e ambientais, avaliar a sustentabilidade da produção de farinha de mandioca. A relevância desta temática é justamente zelar pela vida das pessoas que encontram no campo, sua reprodução social.

\section{METODOLOGIA}

Então, para realização do trabalho, foram coletados dados primários através da aplicação de questionários semiestruturados (YIN, 2015), observações, gravações das conversas e registros fotográficos no local. Os questionários foram constituídos por questões referentes a dinâmica social, econômica e ambiental dos comunitários produtores de farinha de mandioca. Assim, os questionários foram aplicados inicialmente com os líderes da comunidade, que apontaram os comunitários que possuíam casa de farinha em suas residências, sendo protagonistas para a pesquisa, totalizando em 5 (inventário 100\%).

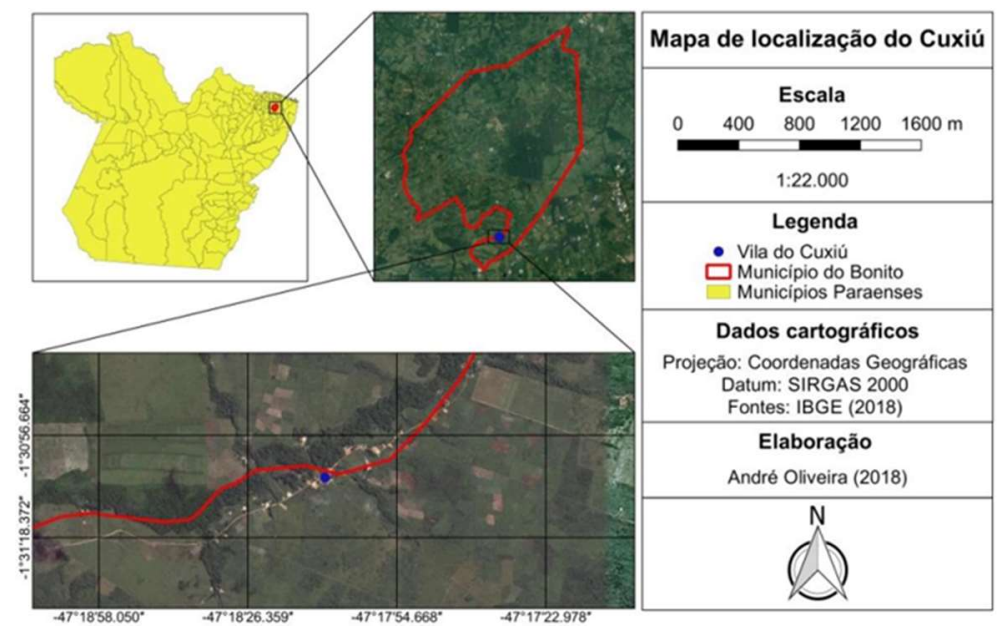

Figura 2: Mapa de localização da Comunidade Quilombola do Cuxiú. Fonte: LIMA et al. (2018).

Os questionários foram aplicados em duas visitas feitas à comunidade, nos dias 01 de Dezembro de 
2018 e 12 de Janeiro de 2019, respectivamente. Os dados secundários foram obtidos através de dados oficias em plataformas online.

A comunidade quilombola do Cuxiú (figura 2) está localizada na Mesorregião Nordeste Paraense, a 25 quilômetros de distância do município de Bonito, fazendo fronteira com Ourém e São Miguel e próximo aos municípios da Microrregião Bragantina, Capanema, Peixe-boi e Santa Maria.

\section{RESULTADOS E DISCUSSÃO}

O plantio da mandioca ocorre em terrenos próximos à casa de farinha ou no próprio quintal, formado por pequenos lotes de terra, em consórcio com outras culturas de espécies como a banana, açaí e mamão que são destinadas ao Programa Nacional de Alimentação Escolar (PNAE) no município sede.

Em relação ao manejo da mandioca, $80 \%$ dos entrevistados (4 moradores) utilizam herbicidas e adubo químico para o manejo da mandioca. Afirmaram ainda que fazem uso de produtos químicos de forma intuitiva, sem informação técnica.

Quadro 1: Uso de produtos químicos na cultura da mandioca.

\begin{tabular}{l|l|l}
\hline Entrevistado & Uso de produtos químicos & Quais? Há quanto tempo? \\
\hline $\mathbf{0 1}$ & Não & Nunca foi necessário; \\
\hline $\mathbf{0 2}$ & $\operatorname{Sim}$ & Herbicida, há 1 ano; \\
\hline $\mathbf{0 3}$ & $\operatorname{Sim}$ & Adubo químico, há 8 anos; \\
\hline $\mathbf{0 4}$ & $\operatorname{Sim}$ & Herbicida e Adubo Químico, há 4 anos; \\
\hline $\mathbf{0 5}$ & $\operatorname{Sim}$ & Herbicida, há 1 ano; \\
\hline
\end{tabular}

Quadro 2: Divisão das atividades da produção de farinha por gênero.

\begin{tabular}{|l|l|l|}
\hline & Gênero & Atividades \\
& Homem & $\begin{array}{l}\text { Plantio } \\
\text { Colheita } \\
\text { Trituração } \\
\text { Prensagem } \\
\text { Esfarelamento } \\
\text { Torração } \\
\text { Empacotamento } \\
\text { Armazenagem }\end{array}$ \\
\hline \\
\hline
\end{tabular}

Os produtores relataram que fazem uso destes produtos para eliminar as ervas daninhas no plantio da mandioca que vem crescendo gradualmente. No entanto, Oliveira Junior (2001) indica que o retorno da 
mandioca à aplicação de herbicidas oscila desde a total seletividade até o comprometimento integral da produção, por conta da fitotoxicidade provocada à cultura.

O quadro 1 demonstra a divisão das atividades por gênero. Além disso, a farinha está presente na mesa todos os dias, o consumo diário é de aproximadamente dois quilos por família, o que faz da casa de farinha um local de interação, onde todos se reúnem com muita frequência.

É possível perceber que atores de diferente faixa-etária estão inseridos no processo, um dos fatores que garantem a disseminação do processo de beneficiamento da farinha entre as gerações, contribuindo para a permanência do homem no campo, além de evidenciar a articulação da agricultura familiar (VAN VELTHEM, 2007).

A farinha de mandioca é produzida nas chamadas 'casas de farinha' com estrutura rústica, fator que reflete na qualidade do produto final que não tem boa visibilidade em relação às condições fitossanitárias. Outro aspecto relevante é a questão dos riscos ergonômicos e da ausência do uso de equipamentos de proteção individual.

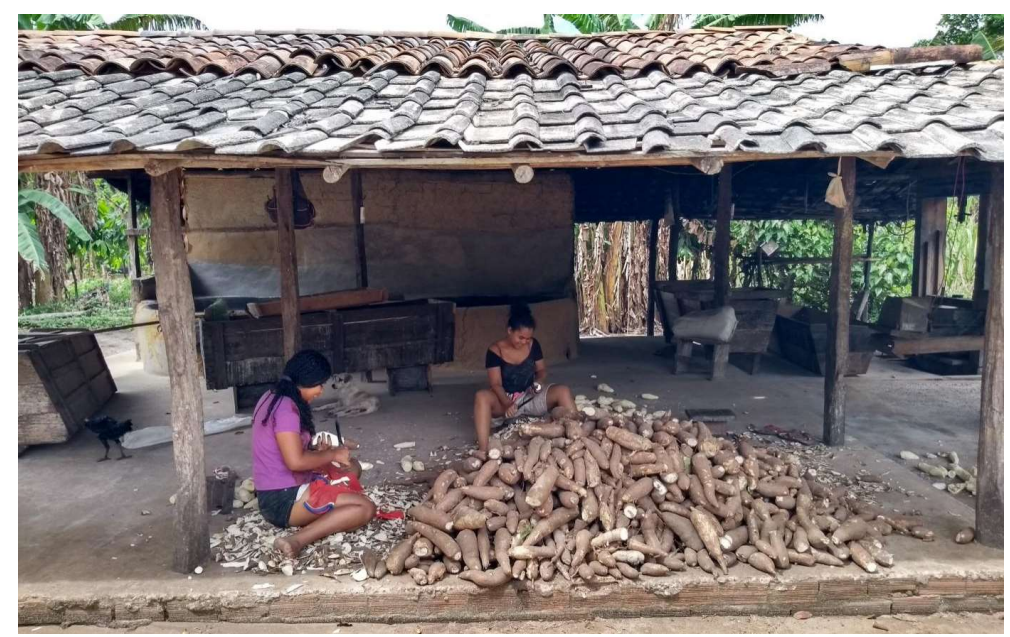

Figura 3: Mulheres conduzindo a etapa de descascamento da raiz da mandioca.

Os resíduos gerados durante o processo são as cascas e a água residual proveniente da lavagem e prensagem da mandioca conhecida como manipueira. As cascas são doadas para uma fazenda vizinha para ser incorporada a ração animal, no entanto é o efluente gerado é descartado diretamente no meio ambiente.

Deste modo, Denardin et al. (2013) aponta alternativas para reutilizar a manipueira. Dentre elas, o simples processo de diluição do líquido que dá origem a biofertilizantes, que favorecerão o enriquecimento do solo, ou ainda como bioherbicida para auxiliar no controle de pragas. Este efluente também se transforma no tucupi, após fermentar por 72 horas e cozer por 10 minutos (CHISTE et al., 2011).

A farinha de mandioca tornou-se o produto com melhor retorno financeiro, que agrega maior renda para os comunitários (LIMA et al., 2018). Atualmente, a comercialização da farinha é feita por meio de atravessadores, pois os moradores não têm condições de se deslocar para vender o produto na cidade.

Assim, a tomada de preço da farinha de mandioca depende exclusivamente do valor exigido pelos atravessadores. Comunitários contam que a saca de farinha já chegou a ser comercializada por $\mathrm{R} \$ 280,00$, e hoje oscila entre $\mathrm{R} \$ 80,00$ e $\mathrm{R} \$ \mathbf{7 0 , 0 0}$ reais. Os comunitários sentem essas alterações, pois o preço atribuído 
ao produto muitas vezes nem chega a pagar a compra de materiais.

\section{CONCLUSÕES}

A participação de moradores de diversas faixa-etárias no processo de produção da farinha de mandioca é um ponto positivo que denota a transferência de saberes para os comunitários mais jovens, garantindo sua sobrevivência e tendo subsídios para permanecer no campo, evitando o êxodo rural, mantendo a cultura pelo hábito alimentar histórico quilombola e amazônica do uso de farinha como ingrediente principal.

Notou-se que os comunitários enfrentam várias dificuldades em relação a comercialização da farinha, tanto em relação as condições de higiene e segurança no trabalho, quanto em comercializar. Daí surge a necessidade de maiores incentivos por meio do poder público em auxiliá-los na aquisição de materiais e na formalização do negócio, evitando a desvalorização do produto e a constante oscilação de preços.

Se torna indispensável ainda, a intervenção de órgãos de assistência e extensão rural para apontar as melhores formas de manejo da cultura da mandioca, diminuindo a aplicação de produtos químicos no plantio e melhores alternativas de destinação dos resíduos.

\section{REFERÊNCIAS}

ARAÚJO, M. J.. Fundamentos de agronegócios. 4 ed. São Paulo: Atlas, 2013.

BARLOW, J.; FONSECA, M. T.; RAMOS, R.; STEIL, L.; PARRY, L.; MARKUSSON, N.; FERREIRA, A.. Queimadas e incêndios florestais na Amazônia brasileira: porque as políticas públicas têm efeito-limitado. Ambiente \& Sociedade. São Paulo, v.20, n.4, p.21, 2017.

BRANDÃO, A.; JORGE, A. L.. Comunidades quilombolas, reconhecimento e proteção social. Vértices, Campos dos Goytacazes, v.14, n.1, p.83-101, 2012.

BRASIL. Constituição da República Federativa do Brasil: texto constitucional promulgado em 5 de outubro de 1988, com as alterações determinadas pelas Emendas Constitucionais de Revisão nos 1 a 6/94, pelas Emendas Constitucionais nos $1 / 92$ a 91/2016 e pelo Decreto Legislativo no 186/2008. Brasília: Senado Federal, 2016.

BRASIL. Decreto n. 6.040, de 07 de fevereiro de 2007. Brasília: DOU, 2007.

CHISTE, R. C.; COHEN, K. O.. Teor de cianeto total e livre nas etapas de processamento do tucupi. Rev. Inst. Adolfo Lutz, v.70, n.1, p.41-46, 2011.

DENARDIN, V. F.; KOMARCHESKI, R.. Sustentabilidade socioambiental da produção de farinha de mandioca em Guaraqueçaba-PR. In: JORNADA DE QUESTÃO AGRÁRIA E DESENVOLVIMENTO, 2. Anais. Curitiba: UFPR, 2013. p.10-16.

FREITAS, C. G.; FARIAS, C. S.; VILPOUX, O. F.. A produção camponesa de farinha de mandioca na Amazônia sul ocidental. Boletim Goiano de Geografia, v.31, n.2, p.29-42, 2011.
IBGE. Instituto Brasileiro de Geografia e Estatística. Produção Agrícola Municipal 2017. Rio de Janeiro: IBGE, 2018.

LIMA, N. J. G.; ARAÚJO, F. C.; MELO JÚNIOR, L. C. M.. Estudo da percepção de impactos ambientais na comunidade quilombola do Cuxiú, Bonito, Pará, Brasil. In: ENEEAMB, 16; FÓRUM LATINO-AMERICANO DE ENGENHARIA E SUSTENTABILIDADE. Anais. Palmas, 2018. p.03-05.

LIMA, R. A.. Tratamento de efluentes líquidos de unidades produtoras de farinha de mandioca. Dissertação (Mestrado) - Universidade Católica de Pernambuco, Recife, 2010.

OLIVEIRA JUNIOR, A. U.. A agricultura camponesa no Brasil. 4 ed. São Paulo: Contexto, 2001.

SEBRAE. Relatório técnico. Manual de referência para casas de farinha. Boas práticas de fabricação, diagnóstico ambiental, saúde e segurança no trabalho, ergonomia, projeto arquitetônico. Maceió: SEBRAE, 2006.

SILVA, A. A. D.; NASCIMENTO, A. C. D. S.; BARBOSA, J. M. P.; LOUREIRO, J. P. B. D.; CASTRO, E. M. D.; RESQUE, A. G. L.. Avaliação da sustentabilidade de agroecossistema familiar de uso comum com a Metodologia MESMIS em uma comunidade quilombola em Ipixuna do Pará. Cadernos de Agroecologia, v.13, n.1, 2018.

SOUZA, S. P. F.; SOUZA, E. B.; QUEIROZ, J. C. B.; JUNIOR, J. D. A. S.. Impactos dos anos climáticos extremos no rendimento da lavoura temporária de mandioca na região rural da metrópole de Belém-Pará. Revista Brasileira de Climatologia, v.21, p.95, 2017. 
VAN VELTHEM, L. H.. Farinha, casas de farinha e objetos familiares em Cruzeiro do Sul (Acre). Revista de

Antropologia, v.50, n.2, p.605-631, 2007.
YIN, R. K.. Estudo de caso: planejamento e métodos. 5 ed. São Paulo: Bookman, 2015.

A CBPC - Companhia Brasileira de Produção Científica (CNPJ: 11.221.422/0001-03) detém os direitos materiais desta publicação. Os direitos referem-se à publicação do trabalho em qualquer parte do mundo, incluindo os direitos às renovações, expansões e disseminações da contribuição, bem como outros direitos subsidiários. Todos os trabalhos publicados eletronicamente poderão posteriormente ser publicados em coletâneas impressas sob coordenação da Sustenere Publishing, da Companhia Brasileira de Produção Científica e seus parceiros autorizados. Os (as) autores (as) preservam os direitos autorais, mas não têm permissão para a publicação da contribuição em outro meio, impresso ou digital, em português ou em tradução. 